\title{
Refractive index sensing based on photonic crystal fiber interferometer structure with up-tapered joints
}

\author{
Yong Zhao, *, Xue-gang $\mathrm{Li}^{1}$, $\mathrm{Lu} \mathrm{Cai}^{1}$, Yang Yang ${ }^{2}$ \\ ${ }^{1}$ College of Information Science and Engineering, Northeastern University, Shenyang 110819, China \\ ${ }^{2}$ Hebei Instruments and Meters Engineering Technology Research Center, Chengde 067000, China \\ *Corresponding author: zhaoyong@ise.neu.edu.cn
}

\begin{abstract}
A simple, ultra compact and highly sensitive photonic crystal fiber interferometer (PCFI) for external refractive index (ERI) sensing was proposed and demonstrated in this paper. The PCFI was formed by splicing photonic crystal fiber (PCF) between two single mode fibers (SMFs) with a slight core-offset. The both joints were up-tapered joints which acted as mode splitter/combiner and were made by fusion tapering technique. The Mach-Zehnder interferometer (MZI) incorporated intermodal interference between core mode and cladding modes of the PCF. When the ERI changed, a RI variation of cladding modes would occur and the output interference spectrum would shift. By measuring the wavelength shift of the interference pattern, temperature-insensitive RI measurement could be achieved. In addition, the refractive index sensing properties with the different PCF diameters were also investigated experimentally. Experimental results showed that RI sensitivity could be up to $252 \mathrm{~nm} / \mathrm{RIU}$ in the refractive index range of 1.333-1.379. And it could be anticipated that RI sensitivity could be improved if the PCF diameter continues to decrease. Meanwhile, the sensor had the advantages of simple structure, small size, high sensitivity, low cost and low temperature sensitivity.
\end{abstract}

Keywords: photonic crystal fiber interferometer refractive index sensor Mach-Zehnder

\section{Introduction}

Refractive index (RI) sensing is an important process in many biological and chemical environment applications. In recent years, RI sensors based optical fiber MZIs have been studied extensively because the advantages they offered, such as small size, immunity to electromagnetic interference, the potential for remote operation, etc. The different types of Mach-Zehnder refractive index sensors have been designed and produced, such as the optical fiber MZI composed by two fiber coupler and optical fibers. But it was complex and easily affected by external environment in the measurement process. Therefore, the in-line optical fiber MZIs which had smaller and simpler structure was proposed. Literatures [1-4] reported a serious of in-line optical fiber MZI sensors based on multimode interference, which was fabricated by splicing a section of multimode fiber between SMFs. However, the interference spectrum had low contrast because the different patterns of energy between the multimode fiber was large, which reduced the measure precision. Moreover, In 2008, Bingtian Zhao proposed new methods of splicing a section of SMF between two SMFs with a slight core offset or tapering the SMF twice for producing In-line MZIs which the interference occurred between the core mode and the cladding modes [5, 6]. This sensor had many advantages, such as good stability, easy fabrication, low cost. However, the 
sensitivity of this sensor only was $27 \mathrm{~nm} / \mathrm{RIU}$ and $33 \mathrm{~nm} / \mathrm{RIU}$, respectively. In addition, the above methods were not perfect because the temperature cross sensitivity issue could not be ignored. As everyone known, PCF had been widely studied because its unique properties especially low temperature sensitivity which was caused by a very low thermal expansion coefficient (TEC) and low thermo-optics coefficient [7]. Recently, investigator illustrated an in-line PCFI that a section of PCF was spliced with single mode fibers (SMFs). When the air holes of the PCF at the splicing regions were fully collapsed during the splicing process, a simple and effective in-line PCFI would be formed [8-11]. However, the melting points were easily to break and the light of the cladding was less, which could cause low extinction ratio. Therefore, the up-taper fusing method have been proposed. In 2013, a Mach-Zehnder interferometer based on multimode fiber core sandwiched between two waist-enlarged fiber tapers was designed to measure refractive index sensing. However, it also was sensitive to the temperature[12].

In this paper, a temperature-insensitive and highly sensitive RI sensor based on in-line PCFI was proposed and demonstrated. The sensing structure was formed by splicing SMF-PCF-SMF with a slight core-offset. And the splicing joints were up-tapered joints through in-line fusion tapering technique.. In addition, in order to further improving the coupling degree between the light field and ERI, the interference arms (i.e. the PCF between the two up-tapered joints) were etched. The fundamental sensing principle of our proposed structure was that the cladding modes of PCF will be excited through the up-tapered joint. After propagation through PCF segment, the excited cladding modes were re-coupled into the second SMF, and a periodic transmission spectrum was generated as a result of the interference between them. The effective RIs and energy leakage of these interference modes could be influenced by the external environment and then caused the interference spectrum shift. At this point, the sensing purpose was implemented.

\section{Operating Principle}

Fig. 1 showed the schematic diagram of the proposed highly sensitive RI sensor based on PCFI. The sensing structure was fabricated by splicing a section of PCF between two SMFs with a slight core offset at two up-taper splicing joints which was formed by in-line fusion tapering technique. Final waists of the joints were enlarged to $145 \mu \mathrm{m}$. The length between the two up-tapered joints was $3 \mathrm{~cm}$, which was the length of the sensing arm. The up-taper fusing point is firmer than common collapsed fusion and it contributed to the uniform distribution of light and excited the cladding modes. Moreover, the purpose of the core-offset is increasing the power of the cladding modes and get better extinction ratio. The first up-taper excited the cladding modes, which were recoupled back and interfered with core mode at the second up-taper after spreading a short distance in PCF. And the light path have been shown in Fig.1. Because of the phase difference between the core and cladding modes, an interference output could be observed and it could be used to measure various environmental parameters.

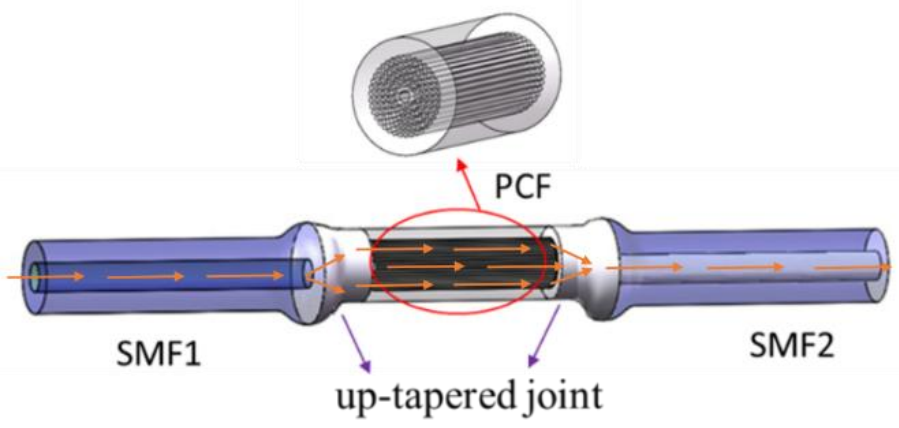

Fig. 1. Schematic diagram of PCFI 
In order to exploring the law of the light propagating in the PCF, electric field distribution was simulated as shown in Fig. 2. In general, when the light propagated in the PCF, the light was limited in the core, i.e. fiber core mode, as shown in Fig. 2(a). However, when the PCF structure changes, such as a dislocation, tapering or collapse, the light would enter in the cladding and the cladding modes could be excited and the electric field was shown in Fig. 2(b), which proved the cladding modes had been excited. About figure 2(b), it was simulated by Rsoft and the launch offset was $1 \mathrm{um}$ (according to the proportion). The launch covered the whole PCF cross section.
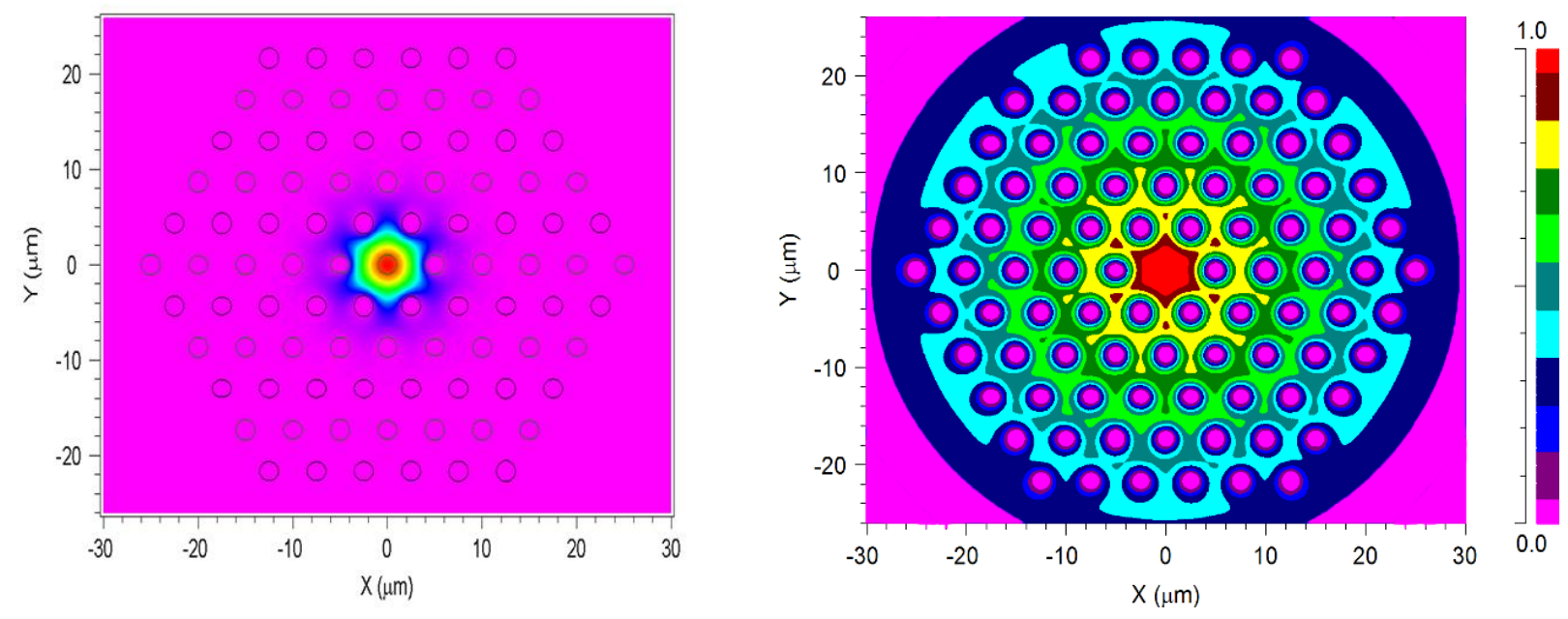

(a) electric field of the fiber core mode. (b) electric field distribution when the cladding modes was excited.

Fig. 2. Simulated electric field distribution of the PCF.

The interference spectrum could be analyzed by using a simple two-mode interference model, which had been widely used in this field to qualitatively analyze the fiber mode interference:

$$
I_{\text {out }}=I_{\text {core }}+I_{\text {cladding }}+2 \sqrt{I_{\text {core }} I_{\text {cladding }}} \cos \delta,
$$

where $I_{\text {core }}$ and $I_{\text {cladding }}$ were light intensities of the core and cladding modes, and $\delta$ were the phase difference between cladding modes and core mode. When the ERI changed, the effective RIs of cladding modes would be influenced but the fundamental mode was unaffected. The RI difference between core mode and cladding modes of the PCF could be expressed as

$$
\Delta n_{0}=n_{\text {core }}^{\text {neff }}-n_{\text {clad }}^{\text {neff }}\left(n_{\text {core }}^{\text {neff }}>n_{\text {clad }}^{\text {neff }}\right),
$$

where $n_{\text {core }}^{\text {eff }}$ and $n_{\text {clad }}^{\text {eff }}$ were effective refractive indices of the core mode and cladding modes. The

phase difference between core mode and cladding modes $\delta_{0}$ could be expressed as

$$
\delta_{0}=\frac{2 \pi L}{\lambda} \times \Delta n_{0}
$$

where $\lambda$ was the centre wavelength of the light. When the $n_{\text {clad }}^{\text {neff }}$ had a small incremental $\Delta n_{1}$ with the ERI and then the total phase different could be obtained by

$$
\delta=\frac{2 \pi L}{\lambda} \times\left(\Delta n_{0}-\Delta n_{1}\right) .
$$

It could be seen from the above formula that when the ERI changed, the $I_{\text {out }}$ would change. 
Therefore, the valley wavelength and transmission loss would vary with ERI changes. Therefore, RI measurement was achieved by measuring the wavelength variations in the spectral response of the interferometer.

\section{Experiments and Results}

For fabricating a PCFI, a commercial single-mode PCF consisted of a solid core surrounded by five rings of air holes was employed. As shown in Fig. 1, such a fiber had a $9 \mu \mathrm{m}$ diameter core, $125 \mu \mathrm{m}$ diameter cladding and five rings of voids that average diameter was $5 \mu \mathrm{m}$ and the average separation between the voids was $7.5 \mu \mathrm{m}$. The experiment system for investigating the RI sensing properties of the sensor was shown in Fig. 3. It consisted of a broadband amplified spontaneous emission source (ASE, wavelength ranging from 1520 to $1570 \mathrm{~nm}$ ), a v-slot (with the tested liquid), the sensing structure and an optical spectrum analyzer (OSA, AQ6370). The resolution of the OSA was set at $0.02 \mathrm{~nm}$ for all experiments and the sensing PCF was placed in the $\mathrm{v}$-slot

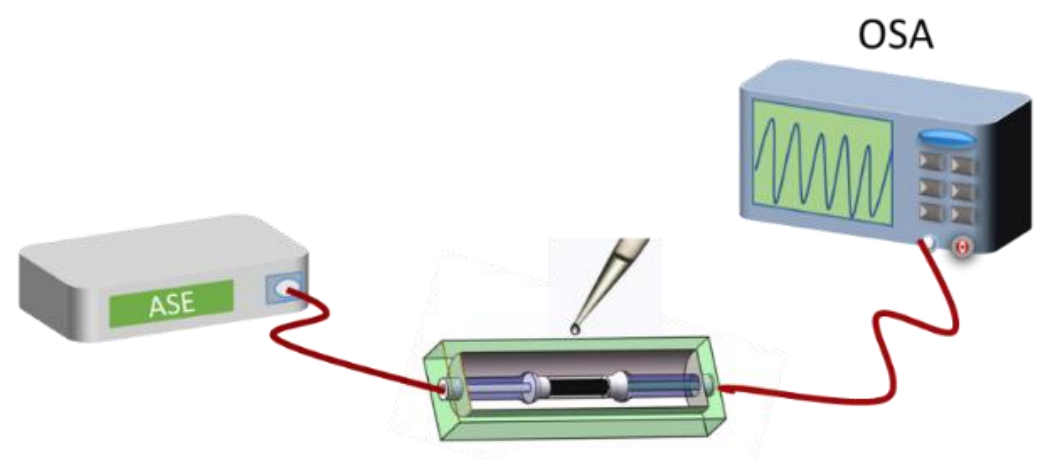

Fig.3 The diagram of experimental system.

In this paper, the sensing structure consisted of SMF-PCF-SMF with two special up-tapered joints, as shown in Fig.1, and the optical microscope micrograph of the PCF was shown in Fig. 4(a), which showed that the diameter of fiber had been increased to the $145 \mu \mathrm{m}$ at the up-tapered joints acting the beam splitter/combiner. The offset can be controlled by fusion technique and the control precision can reach to 0.1 um in our experiment. The up-taper section was made by controlling forward distance and discharge strength of the fusion splicer. The longer forward distance formed wider up-taper. The input optical signal was split into two optical paths at first coupling point, along the core and the cladding of the PCF, respectively, and then they were re-combined together at the second coupling point. When a broadband light source was connected to the input, an interference output, as shown in Fig. 5(a), could be observed. With the increase of liquid RI, the spectrum will move to the right, as shown in Fig. 5(a). By measuring the wavelength shift of one transmission dip with an optical spectrum analyzer (OSA), and then ERI could be determined. For water, i.e. the RI is 1.333, one of the transmission minimums occured at $1556.24 \mathrm{~nm}$ and shifted to longer wavelength with the increase of liquid RI. When the RI was increased to 1.379 , a $6.44 \mathrm{~nm}$ wavelength shift of the transmission minimum was measured, as shown in Fig. 5(a), where the high RI of the liquid would cause a change in the effective indices of the cladding modes, leading to a wavelength shift of the resultant spectrum. It is worth mentioning that the spectra were not uniform and smooth, which caused by the multiple modes were excited and superimposed in the interference spectrum. As a result, the crosstalk problem could be introduced and the result exhibit poor linearity under changes in external refractive index.

In order to further improving the sensitivity, the sensing PCF diameter was decreased by a simple hydrofluoric acid (HF) etching method. When the PCF diameter decreased, the interaction between the 
evanescent waves of the cladding modes and the ambient environment around the fiber will be enhanced, which meant that the RI resolution of the sensor will be improved. The optical microscope micrograph of the PCF after etching were shown in Fig. 4(b) and Fig. 4(c).
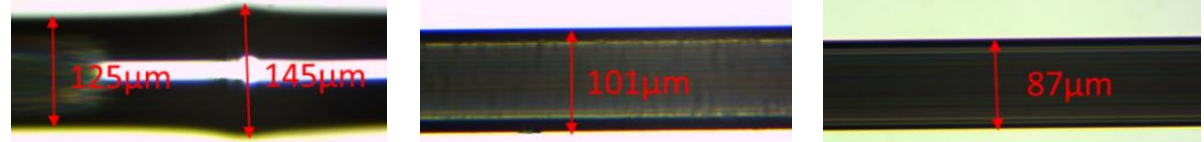
(a) PCF-not-etching
(b) PCF-etching diameter to $101 \mu \mathrm{m}$
(c) PCF-etching diameter to $89 \mu \mathrm{m}$

Fig.4 Different diameter PCFs under the microscope

By the same token, the spectrum with different ERI were recorded, respectively, as shown in Fig.5 (b) and Fig. 5(c). And the fitting curves between the characteristic wavelengths and ERI were shown in Fig. 5(d), which showed spectrum of the different diameter PCF shifted with the different RI of the surrounding sodium chloride solution. As shown in Figure 4(d), the RI sensitivity of sensor was $140.8 \mathrm{~nm} / \mathrm{RIU}$ with no-etched PCF and it increased as the diameter PCF decrease. When the diameter of PCF was $87 \mu \mathrm{m}$, the sensitivity could be up to $252 \mathrm{~nm} / \mathrm{RIU}$. In addition, the wavelength resolution of OSA used in experiment were 20pm, so the resolution of measuring RI was $8 \times 10^{-5}$. Predictably, the sensitivity can be still increased with the PCF diameter decreases. Table 1 was the measurement error between the measurement data and actual data, which showed small errors.

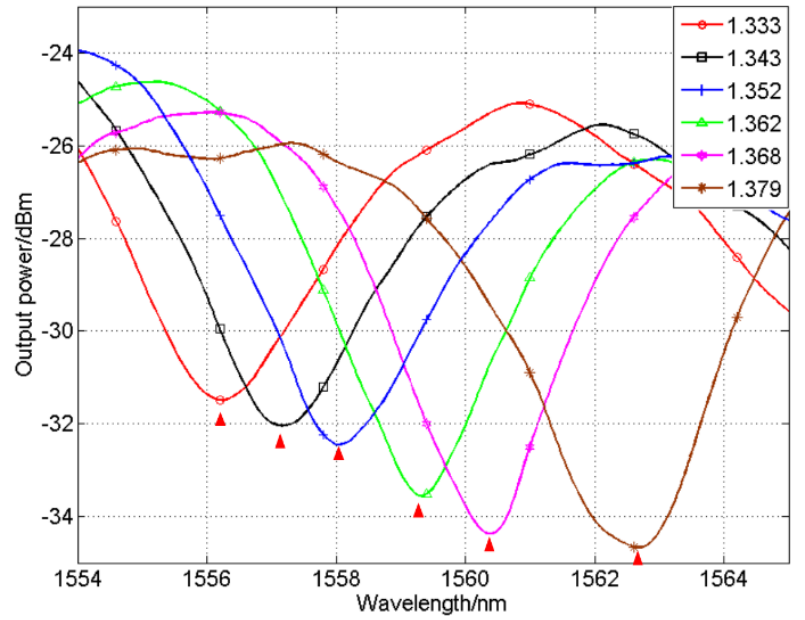

(a) spectra of PCFI with $125 \mu \mathrm{m}$ PCF

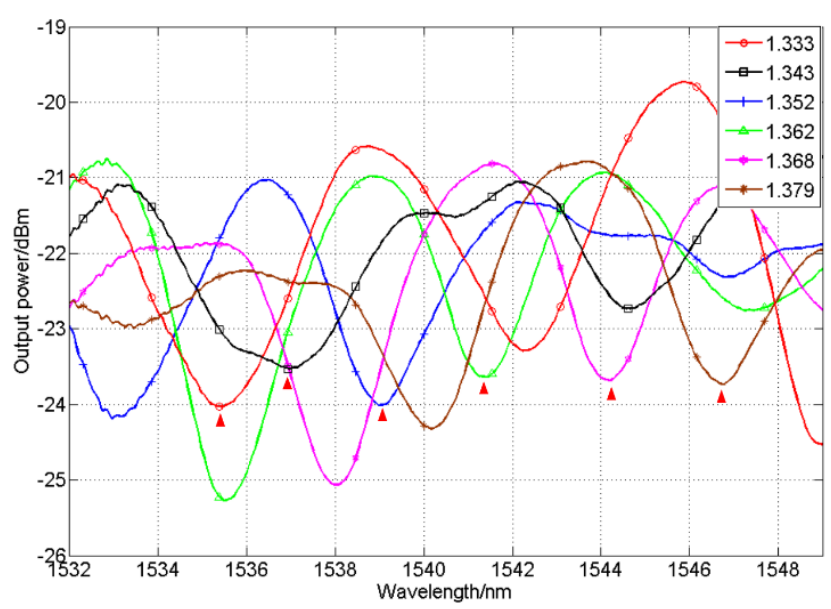

(b) spectra of PCFI with $87 \mu \mathrm{m}$ PCF

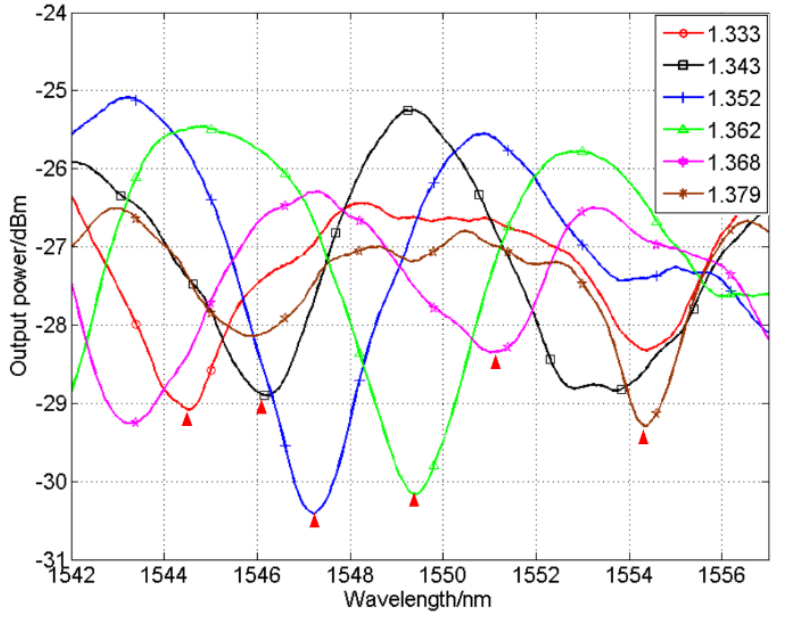

(b) spectra of PCFI with $110 \mu \mathrm{m}$ PCF

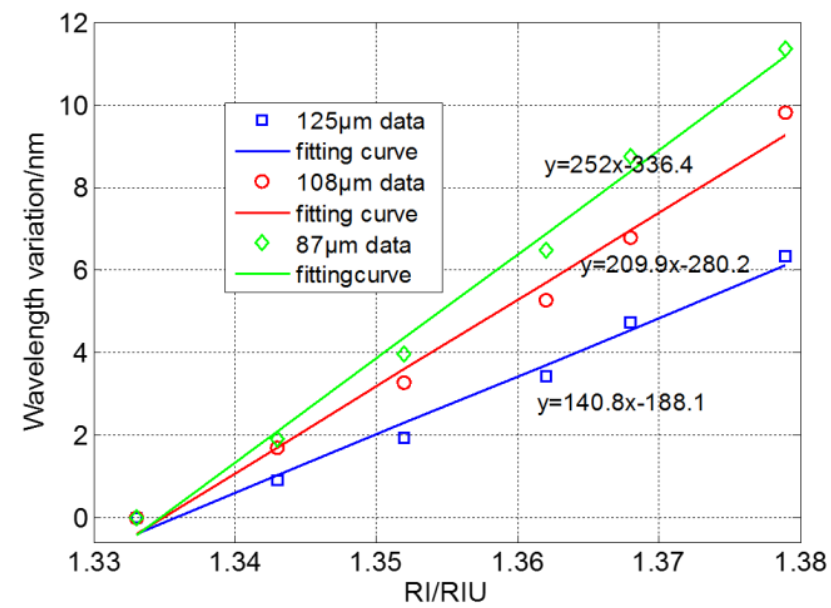

(d) Fitting curve between characteristic wavelength and ERI

Fig.5 spectra of the different diameter PCF with different ERI and fitting curve between characteristic wavelength and ERI. 
Tab.1 The error between the actual data and measurement data.

\begin{tabular}{ccc}
\hline The actual RI & The measurement RI & Measurement error \\
\hline 1.333 & 1.3349 & 0.0016 \\
1.343 & 1.3425 & -0.0005 \\
1.352 & 1.3507 & -0.0013 \\
1.362 & 1.3607 & 0.0013 \\
1.368 & 1.3697 & 0.0017 \\
1.379 & 1.3799 & 0.0009 \\
\hline
\end{tabular}

Considering temperature, the silica-based sensors exhibit low temperature sensitivity due to a very low thermal expansion coefficient (TEC) of about $5.5 \times 10^{-7} /{ }^{\circ} \mathrm{C}$ and low thermo-optics coefficient (TOC) of about $5.5 \times 10^{-6} /{ }^{\circ} \mathrm{C}$ [7], which have been further validated by experiment in this paper. Fig. 5 showed the measured transmission spectra under different temperatures. It could be seen that the interference pattern under different temperatures would blue shift and the temperature sensitivity only was $2.96 \mathrm{pm} /{ }^{\circ} \mathrm{C}$. This meant the equivalent RI change only was $1.178 \times 10^{-7}$ when temperature changed $1^{\circ} \mathrm{C}$, which could be ignored compared with the RI sensitivity.

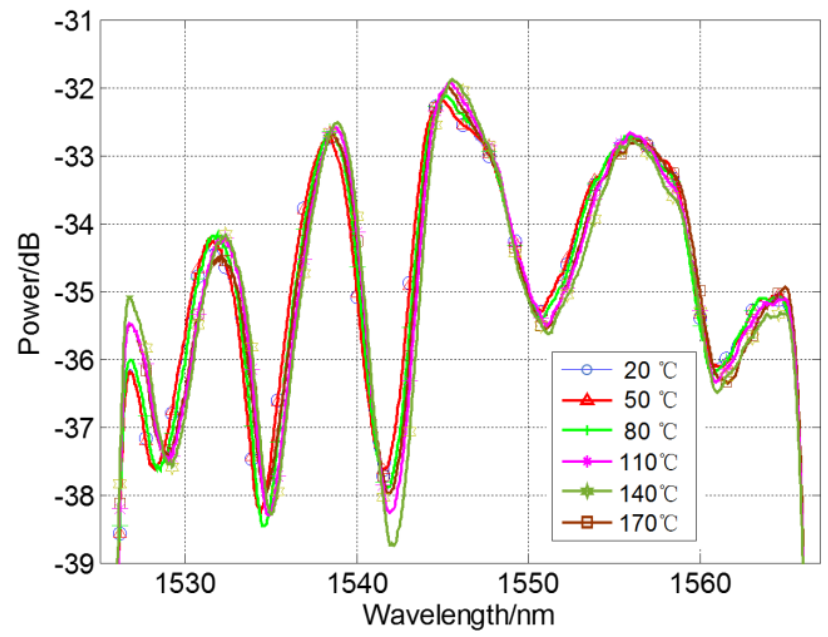

(a) spectra with the different temperature

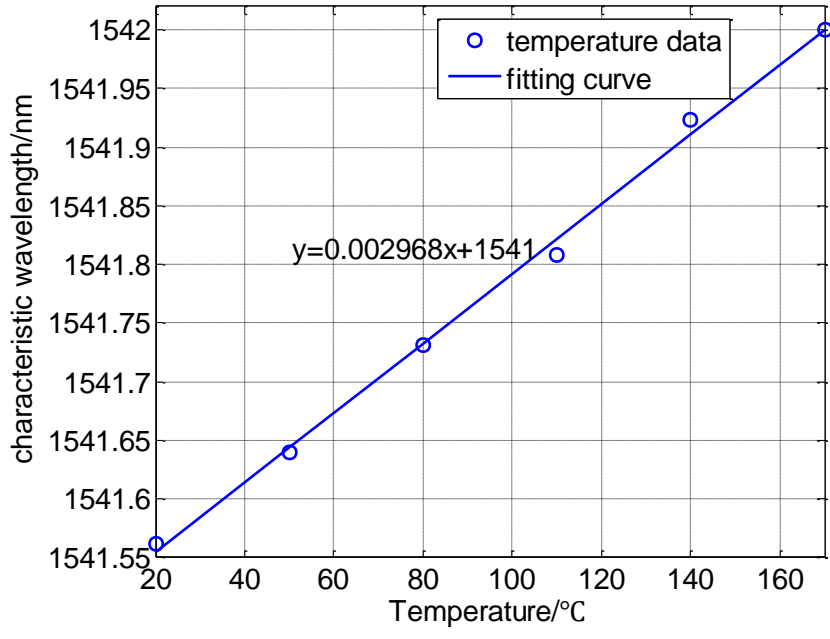

(b) Fitting curve between the temperature and wavelength

Fig.6 spectra with the different temperature and the fitting curve

\section{Conclusions}

A new, simple and highly sensitive RI sensor based on in-line PCFI which was formed by up-taper splicing a piece of PCF between two SMFs with a slight core offset was proposed and demonstrated in this paper. The up-taper joints acted as mode splitter and combiner. Experimental results showed that the RI sensitivity could be up $252 \mathrm{~nm} / \mathrm{RIU}$ with the range of $1.333 \sim 1.379$ and the resolution of 
measuring RI was $8 \times 10^{-5}$. Predictably, the sensitivity can still increase with the PCF diameter decreases. Moreover, the RI sensor kept low temperature sensitivity that only was $2.96 \mathrm{pm} /{ }^{\circ} \mathrm{C}$, which could be ignored compared with the RI sensitivity. Therefore, temperature insensitive RI measurement has achieved by measuring the wavelength variations in the spectral response of the interferometer.

\section{Acknowledgments}

This work was supported in part by the National Science Foundation for Distinguished Young Scholars of China under Grant 61425003, the National Natural Science Foundation of China under Grant 61273059, the Fundamental Research Funds for the Central Universities under Grant N140404021 and N130104002, and State Key Laboratory of Synthetical Automation for Process Industries under Grant $2013 Z C X 09$.

\section{References}

[1] S. Silva, E.G.P. Pachon, M.A.R. Franco, et al., Curvature and temperature discrimination using multimode interference fiber optic structures - a proof of concept[J]. Journal of Lightwave Technology, 2012, 30(23): 3569-3575.

[2] S. Silva, O. Frazão, J. Viegas, et al., Temperature and strain-independent curvature sensor based on a singlemode/multimode fiber optic structure[J]. Measurement Science and Technology, 2011, 22(8): 085201.

[3] E. Li, X. Wang, C. Zhang, Fiber-optic temperature sensor based on interference of selective higher-order modes[J]. Applied Physics Letters, 2006, 89(9): 091119-091119-3.

[4] J. Wo, Q. Sun, H. Liu, et al., Sensitivity-enhanced fiber optic temperature sensor with strain response suppression[J]. Optical Fiber Technology, 2013, 19(4): 289-292.

[5] Z. Tian, S. Yam, H. Loock, Single-mode fiber refractive index sensor based on core-offset attenuators[J]. Photonics Technology Letters, 2008, 20(16): 1387-1389.

[6] Z. Tian, S. Yam, In-line single-mode optical fiber interferometric refractive index sensors[J], Journal of Lightwave Technology, 2009, 27(13) 2296-2306.

[7] H. Jui-Ming, H. Jing-Shyang, Fiber-optic Michelson interferometer with high sensitivity based on a liquid-filled photonic crystal fiber[J], Optics Communications, 2014, 331: 348-352.

[8] H. Y. Choi, M. J. Kim, B. H. Lee. All-fiber Mach-Zehnder type interferometers formed in photonic crystal fiber[J]. Optics Express, 2007, 15(9): 5711-5720.

[9] A. S. Href, R. Amezcua-Correa, J. P. Carvalho, et al. Modal interferometer based on hollow-core photonic crystal fiber for strain and temperature measurement[J]. Optics express, 2009, 17(21): 18669-18675.

[10] X. Bai, D. Fan, S. Wang, et al. Strain sensor based on fiber ring cavity laser with photonic crystal fiber in-line Mach-Zehnder interferometer[J]. IEEE Photonics Journal, 2014, 6(4): 6801608.

[11] T. Li, X. Dong, C. C. Chan, et al. Simultaneous strain and temperature measurement based on a photonic crystal fiber modal-interference interacting with a long period fiber grating[J]. Optics Communications, 2012, 285(24): 4874-4877.

[12] M. Shao, X. Qiao, H. Fu, et al. High sensitivity refractive index sensing of Mach-Zehnder interferometer based on multimode fiber core sandwiched between two waist-enlarged fiber tapers[J]. Optics Communications, 2013, 311: 359-363. 